\title{
"Cast Iron - A Predictable Material" 25 Years of Modeling the Manufacture, Structures and Properties of Cast Iron
}

\author{
Jörg C. Sturm ${ }^{1, a^{*}}$ and Wilfried Schäfer ${ }^{1, b}$ \\ ${ }^{1}$ MAGMA Gießereitechnologie GmbH, Kackertstrasse 11, 52072 Aachen, Germany \\ aJC.Sturm@magmasoft.de, bW.Schaefer@magmasoft.de \\ Dedicated to Prof. Ingvar L. Svensson
}

\begin{abstract}
Keywords: Casting Process Simulation, Micromodelling, Defects, Structures, Properties, DOE, Virtual Optimization, Robust Processes
\end{abstract}

\begin{abstract}
During the last 25 years, casting process simulation has developed from predicting hot spots and solidification paths to an integral assessment and optimization tool for foundries for the entire manufacturing route of castings. Modeling cast irons has always been a special challenge due to the strong interdependency between the alloy composition, applied metallurgy and metal treatment with the solidification, phases and structures which form and the resulting properties of the material.
\end{abstract}

Supporting the risering of the casting is still one of the most important uses of casting process simulation. Different feeding behaviors and self-feeding capabilities of cast irons need to be considered to provide a defect free casting. To be able to quantitatively predict these defects, already in the early 1990's solidification simulation was coupled to so-called micromodeling. This allowed the consideration of major controlling parameters in the foundry, such as the influence of alloying elements, melting practice and metallurgy, on the special shrinkage and solidification behavior of cast irons. As an additional benefit, the prediction of local structures, phases and ultimately the local mechanical properties of cast irons was available, to assess casting quality in the foundry but also to make use of this quantitative information during design of the casting.

Today, casting quality means more than soundness. A comprehensive list of additional quality issues such as dross or sand inclusions and thermally driven stresses and distortion in castings and cores can be modeled. Cracks in castings can be assessed, as well as the reduction of casting stresses during heat treatment. Increasing demands on material performance has led to increased property requirements for cast irons. The demand for reliable information for new alloys and materials such as CGI, ADI or high-Si ductile cast iron has strongly grown and was addressed by extended modeling capabilities.

All this quantitative information about the material's performance is most valuable if it can be used during casting design. The transfer of local properties into the designer's world, to predict local properties such as fatigue strength and durability as a function of the entire manufacturing route, will increase the trust in this old but highly innovative material and will open new opportunities for cast iron in the future.

In each case, the basis for extended modeling and simulation capabilities has been to first gain a fundamental understanding of the formation mechanisms. This requires smart experimentation coupled with the skill to turn empirical and experimental knowledge into quantitative physical models. This paper provides an overview of 25 years of cast iron modeling, which is strongly linked to the achievements and the lifetime dedication of Prof. Ingvar Svensson to cast iron. He has been a pioneer in gaining quantitative understanding to predict cast iron using a computer. The paper will sketch some selected highlights of his work and will provide an outlook to current and future demands on integrated cast iron research to continue to make cast iron a predictable material.

The first part of this paper introduces the challenges and discusses the requirements to model the material cast iron from process to structures to local mechanical properties. In the second part there are presented the recent achievements in the integration of local casting properties into the design 
chain and there is introduced the future of moving from casting process simulation to virtual DOE and autonomous optimization.

\section{Introduction}

The metal casting industry has always tried to balance both technical and commercial needs, maintaining engineering capabilities, ensuring efficient operations and protecting business profitability. Business at its root has not changed, but the way that business is carried out is changing. There is a tremendous decentralization underway. Casting clients are outsourcing responsibilities and increasingly globalizing. As such, the technical requirements and breadth of responsibility placed on cast iron foundries have become even more demanding. This places additional requirements on our engineering resources, and challenges us to think about new ways to shorten lead times, reduce total costs, and technically interact with clients more effectively.

With the upcoming capabilities of CAE technologies including fast evolving casting process simulation and new computer based component design tools, it is possible for foundrymen and designers to work together concurrently, to optimize both component design and casting process parameters. Through these engineering efforts, foundrymen can assure the sustainability and growth of their businesses while maintaining a sizable technical edge over competition. Quantitative results provided by casting process simulation help designers to understand the impact of the process on the performance of castings in use.

To meet today's specifications in making cast iron, ductile iron, compacted graphite iron or even austempered ductile iron requires a profound understanding of the material and the process robustness. Here, casting process simulation has been extremely instrumental. During the recent more than two decades, the technology of simulating the casting process and predicting the resulting material properties has become helpful in two ways: First, making the mold as a black box transparent for the foundry specialist, helping him to understand the root causes of possible problems prior to the first casting. Secondly, developing virtual simulation tools for the casting process requires a profound and quantitative understanding of the impacts of physics, metallurgy and chemistry as such. This has changed the empirically driven process substantially into a first principle based and reliable manufacturing process, Fig. 1.

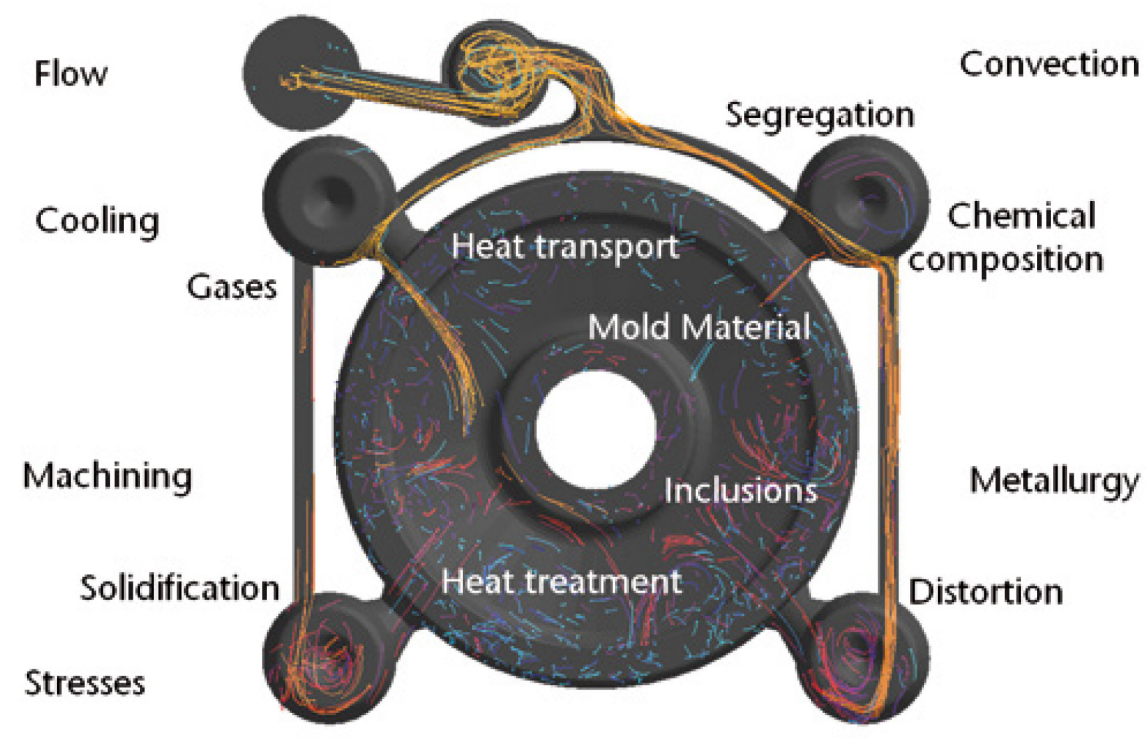

Fig. 1: A challenging task: Simulating the casting process to predict component properties. The biggest benefit of the casting process is its ability to perform many tasks at the same time. However, it is also its biggest drawback, as many process parameters are linked to each other and have to be considered simultaneously. 
For many cast iron foundries, casting process simulation is used daily as a standard tool to assess gating and risering and to predict feeding. It has become an instrument in quality systems and process optimization. State-of-the art simulation tools consider the special material behavior of cast irons with respect to its alloy composition, melting practice, and metallurgy [1]-[4].

After designing the gating system and the pattern lay-out, a first complete simulation of the entire process can be done. The basis for the simulation is the calculation of different phases and their amounts for the entire solidification of the casting. This allows the determination of the local sum of shrinkage as a function of the currently present contracting (liquid, austenite and cementite) or expanding (graphite) phases, and its compensation through feeding from a riser.

Once isolated regions are formed which can no longer be fed, the total feeding is a sum of remaining liquid and austenitic shrinkage, and local graphite expansion. Additionally, mold stability and mold dilatation must be considered to take the self-feeding effects into account. Only this micromodelling approach enables the prediction of porosity in cast iron, Fig. 2.

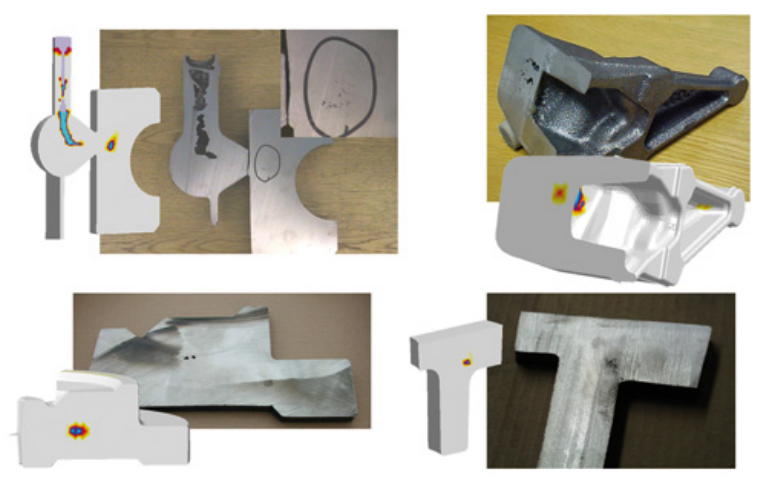

Fig. 2: Shrinkage prediction and reality. Examples displaying the accuracy of shrinkage prediction for different cast iron castings.

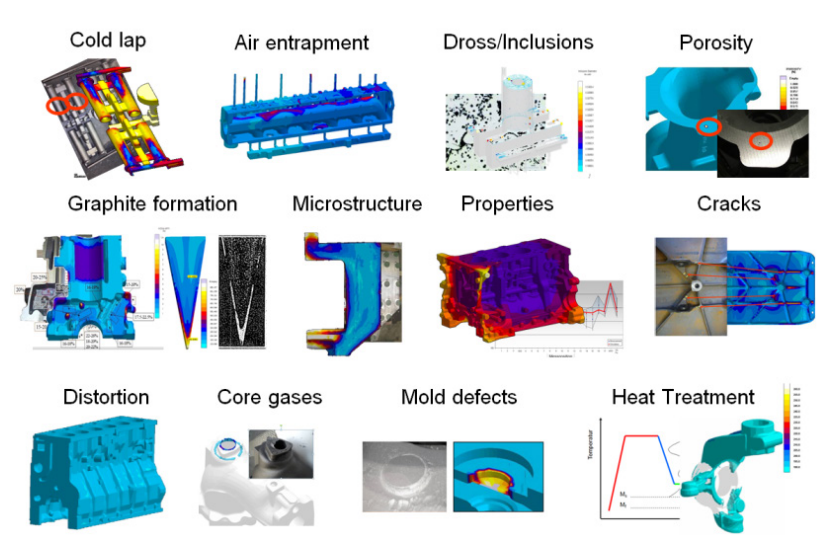

Fig. 3: Cast iron processes, defects and properties addressable by state-of-the art casting process simulation.

The current development efforts go far beyond the evaluation of solidification and feeding. One focus is related to the prediction of complex defects resulting from an interaction of metallurgy and process. A second development aspect is focused on the modelling and prediction of the entire manufacturing route. All this is required to get to the ultimate goal of casting process simulation: The prediction of local casting properties to assess the component's design, the complete casting and process engineering, and their economic impact on the profitability of cast iron castings, Fig. 3.

\section{Requirements for Benefiting from Casting Process Simulation of Cast Iron}

The melting and metallurgical practice applied has a decisive impact on the casting integrity. This is especially true for cast iron components, in which the metallurgical processing is decisive for the ultimate casting structures and properties. Only if casting process simulation is capable of considering the impact of alloying and metallurgy, can cast structures can be predicted locally.

Supporting the feeding related layout of the casting is still one of the most important uses of casting process simulation. Depending on the alloy poured, different feeding behaviors and selffeeding capabilities need to be considered to provide a defect free casting. Therefore, it is not enough to base the prediction of shrinkage defects solely on hot spots derived from temperature fields, but it is also necessary to be able to quantitatively predict them. Solidification simulation had to be combined with density and mass transport calculations in order to evaluate the impact of the solidification morphology on the feeding behavior, as well as to consider alloy dependent feeding ranges. This is accomplished through the description of temperature dependent thermo-physical properties. 
The special feeding behavior of cast irons and their strong dependency of solidification behavior on metallurgy mean that a macroscopic hot spot prediction is not sufficient to asses the methoding of cast iron castings. In ductile iron, big hot spots mostly result in a perfect precipitation of the graphite and hence in a sound casting. On the other hand, small hot spots occurring early during solidification may lead to strong shrinkage due to austenite contraction and suppression of graphite.

To be able to predict the soundness of cast iron based on the real local shrinkage and expansion of the cast material, the program has to be capable of considering the kinetics of the phases being formed during the entire solidification path individually. For cast iron, this means taking into account the effects of all alloying components, and additionally the applied inoculation and melting practice and metallurgy.

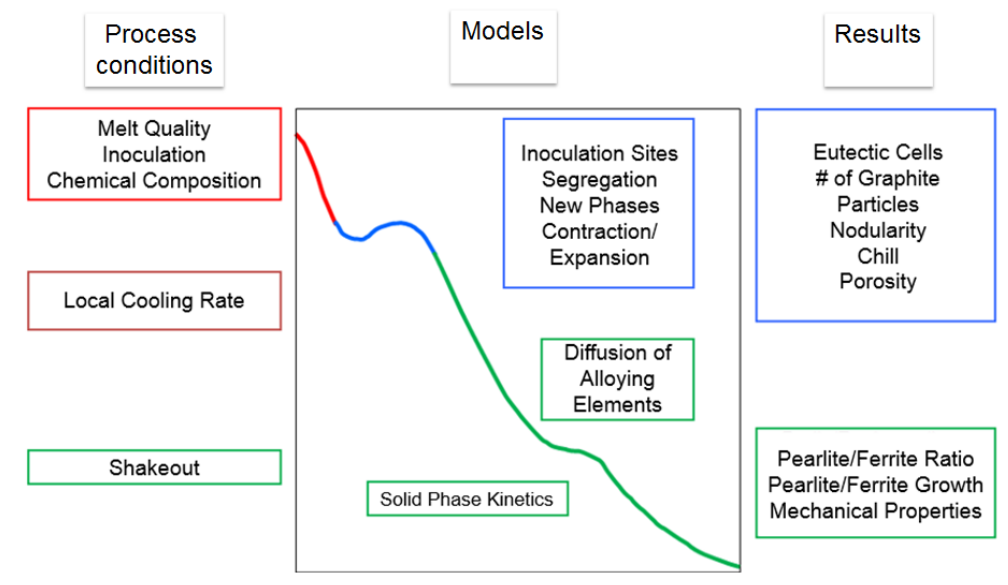

Fig. 4: Simulation of cast iron microstructure under consideration of input parameters, utilized models and available results.

Every foundry specialist makes use of inoculation and alloy composition to avoid chill effects or eutectic cementite. These influences are superimposed by the local cooling conditions. A pure simulation of macroscopic heat flow cannot take this coupled interaction into account. Therefore, so called microstructure models, which predict the amount of new phases based on the above described interactions for any location within the casting at any time, are applied, Fig. 4 [5, 6].

The different capabilities of both models are best evaluated using "simulated" cooling curves. Whereas in macroscopic thermal models the material (thermo-physical) properties are fixed for the used alloy, in a micromodel these properties are determined at each time step and for every point in the casting as a function of the current phase formation. This influences the release of latent heat, and finally the shape of local cooling curves, Fig. 5. Supercooling, recalescense and growth temperatures, which are dependent on local metallurgical and thermal conditions, are a result of the simulations. In the same way that a real cooling curve is used as a measure for the melt quality, the „simulated cooling curve“ is a proof for the quality of the used models. Knowing the actual state of precipitating graphite, austenite and cementite phases at any point, feeding and shrinkage can be predicted locally. 

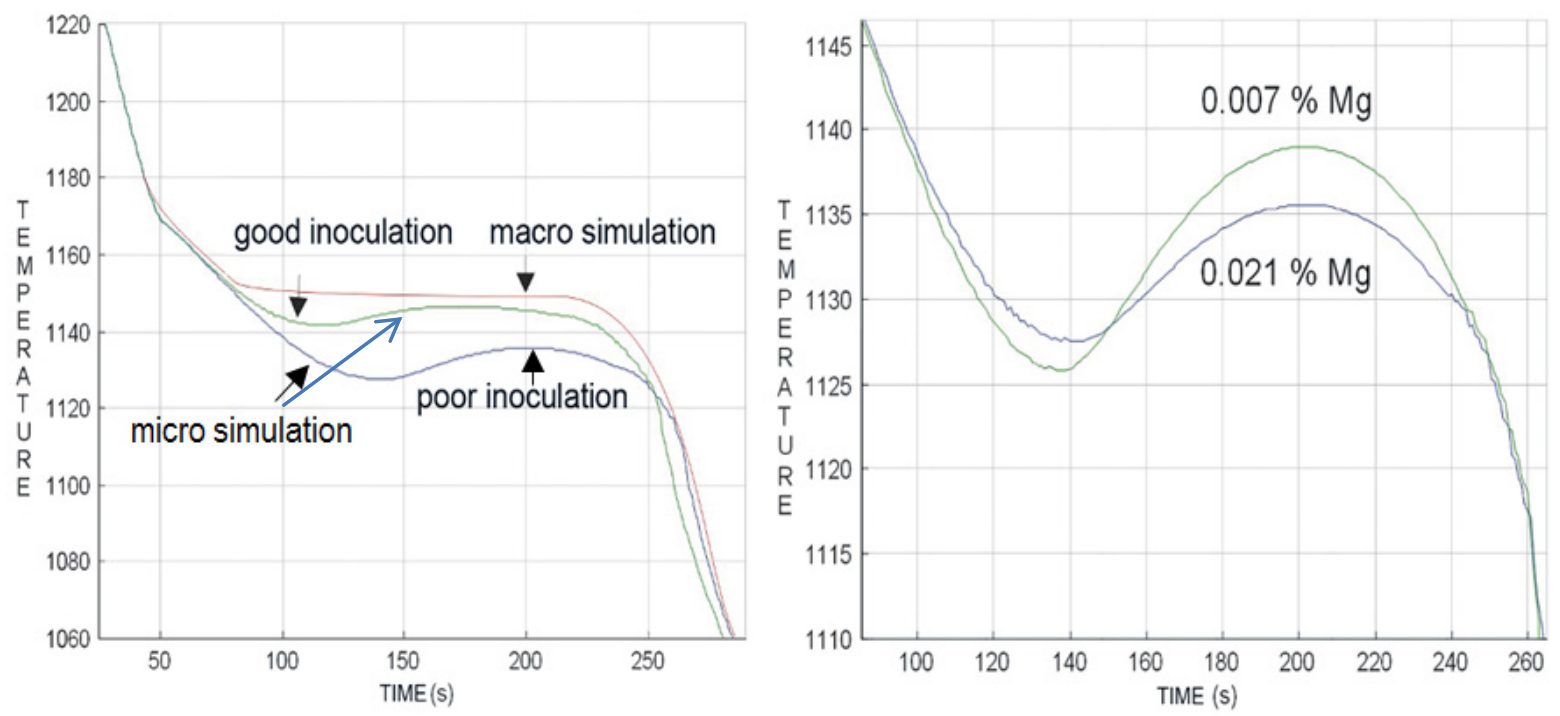

Fig. 5: Sensitivity of cast iron micromodels to the applied metal treatment. The figures show the differences between macroscopic and microscopic simulation (micromodelling), using simulated cooling curves. While the use of macroscopic heat transfer equations only modifies the shape of the cooling curve due to the released latent heat, micromodelling also considers the impact of different inoculation conditions (left). Even composition changes (i. e. change of effective $\mathrm{Mg}$ content between $0.007 \%$ and $0.021 \%$ ) modify the calculated undercooling, recalescense, and growth temperature (right).

\section{Simulation Predicts Microstructures in Cast Iron}

The simulation of individual phases as a function of metallurgy, melting, and inoculation practice also allows a prediction of microstructures after solidification (nodule count/number of eutectic cells, amount of grey/white solidification, and amount of austenite/eutectic graphite), Fig. 6 and 7. Through calculation of the further cooling and local segregation down to the solid-state reaction, the local phase distribution of the matrix (ferrite/pearlite distribution, coarseness of pearlite) can be assessed quantitatively (see also [7-11]). This is important information for the quality systems of foundries, Fig. 8.

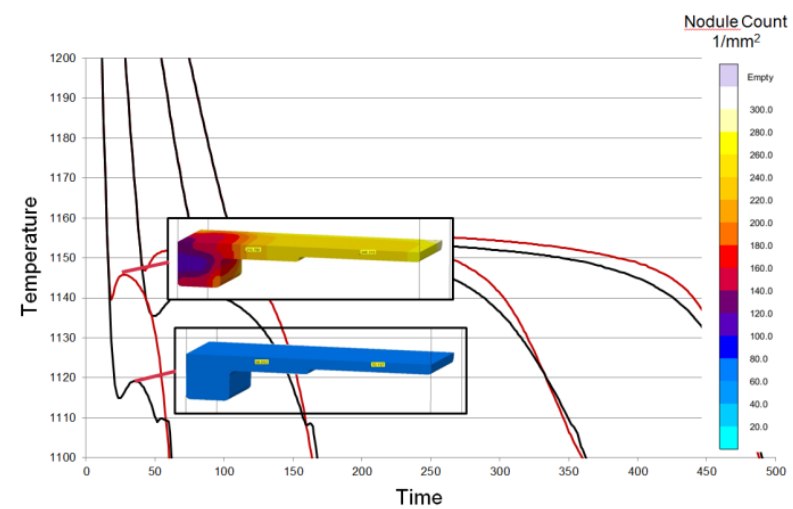

Fig. 6: Simulated cooling curves and respective nodule count maps for a staircase casting. The results show the impact of different inoculation conditions and local cooling rates on the solidification.

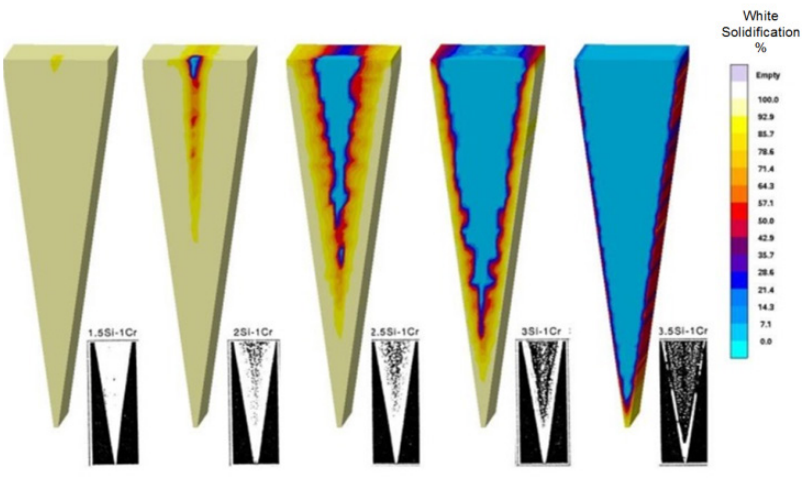

Fig. 7: Simulating the influence of alloying elements on the microstructure. The transition of grey to white solidification in wedge test samples as a function of alloying elements in comparison to the real microstructure. 

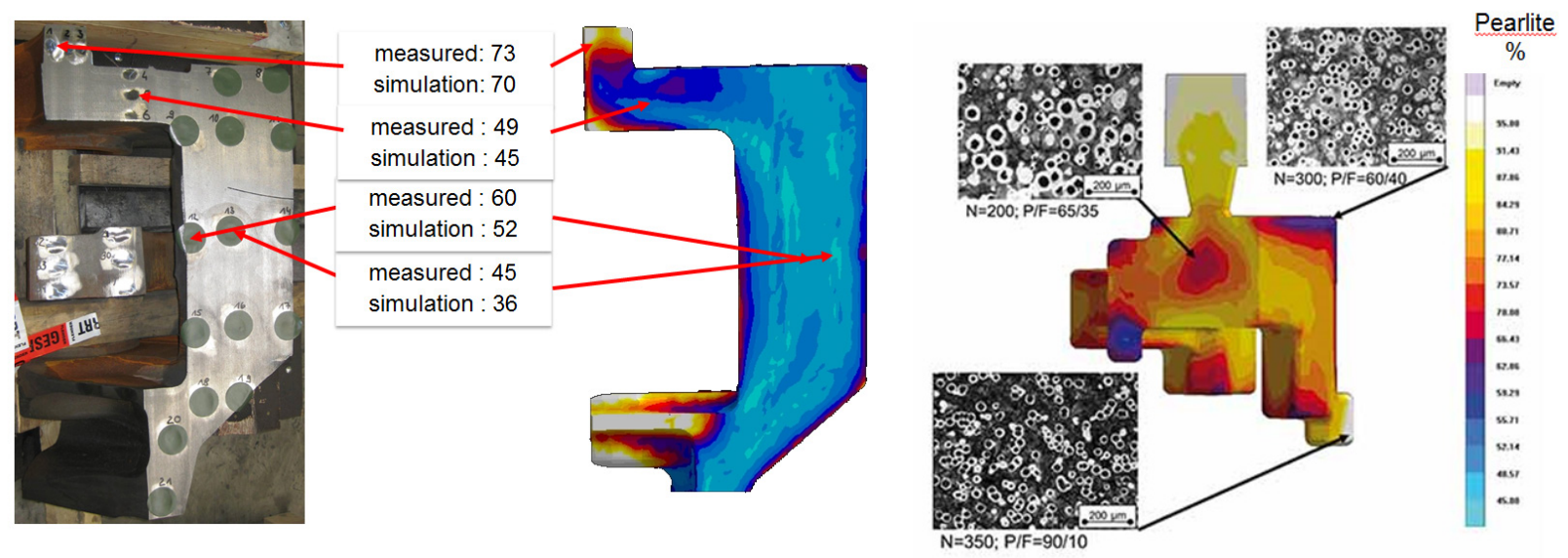

Fig. 8: Assessment of microstructures and mechanical properties for ductile iron. Due to the consideration of nucleation, phase distribution, segregation of alloying elements, and local cooling during solid state reactions, the local nodule count (left) and ferrite/pearlite distribution (right) can be predicted quantitatively with good agreement to real findings [12, 13]

Micromodelling also allows predicting the transition of different graphite morphologies (e. g. Aand D-type graphite and transition from ductile to compacted graphite morphology) as a function of the applied metallurgy, the alloy composition, and the local cooling conditions. Fig. 9 shows the predicted nodularity distribution in an engine block test casting.
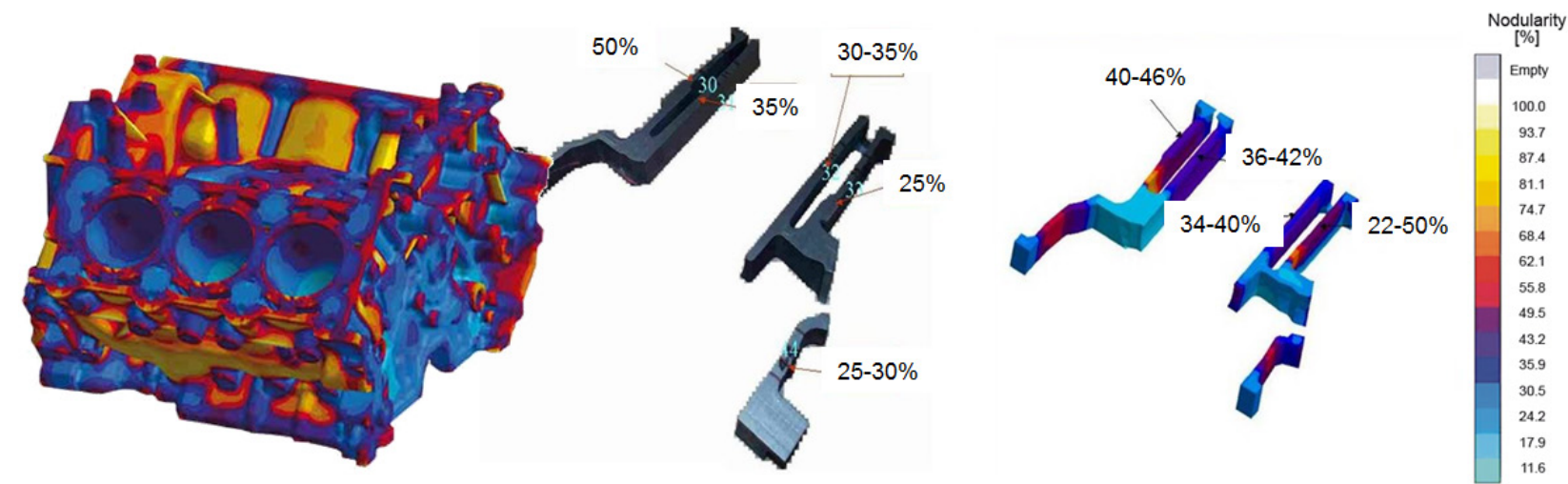

Fig. 9: Simulating local nodularity as a function of alloy, metallurgy, and cooling conditions. Simulated nodularity values are compared with measured nodularity $[14,15]$.

\section{Predicting Mechanical Properties}

The quantitative knowledge about local phases and microstructure allows the prediction of local mechanical properties for the entire casting (tensile strength, hardness, yield strength, elongation and Young's modulus), Fig. 10 [17]-[20]. This prediction is based on the assessment of the local structures or precipitated phases.

Further developments have been made to couple local structure and defect predictions to static and dynamic properties, which can be used for quality inspection within the foundry or as input parameters for safe and lightweight design, Fig. 11 and Fig. 12. 


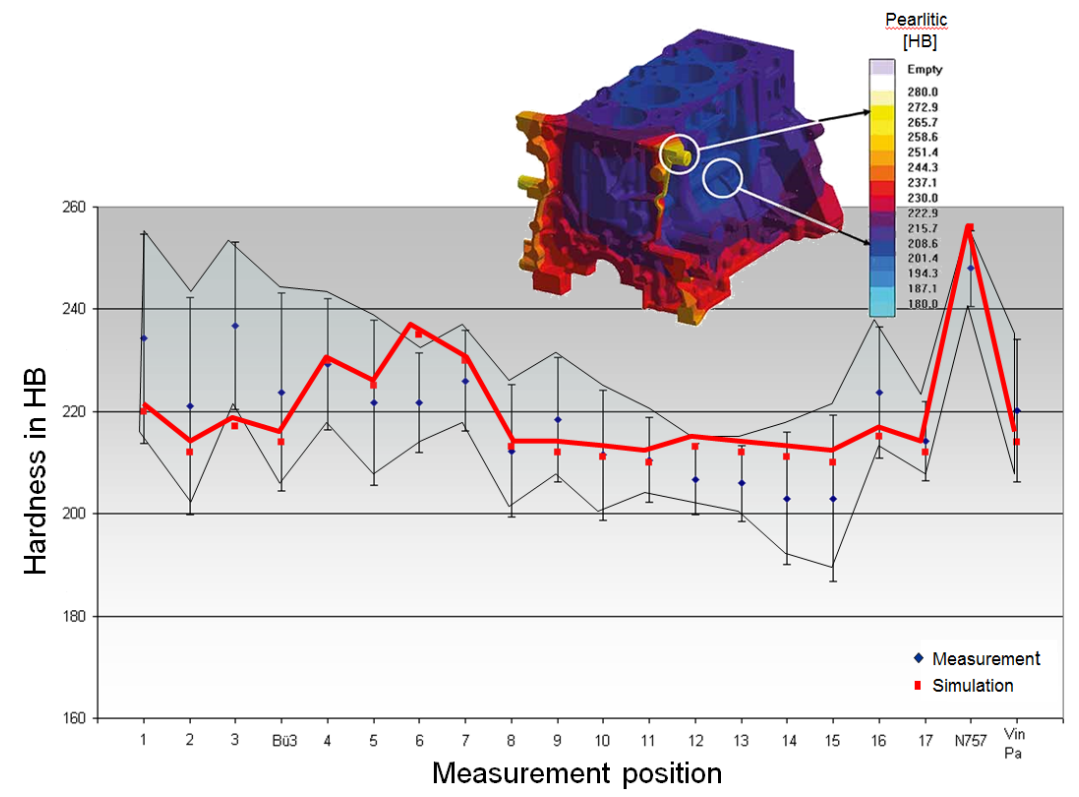

Fig. 10: Comparison of simulated and measured hardness values for a grey cast iron engine block [16].

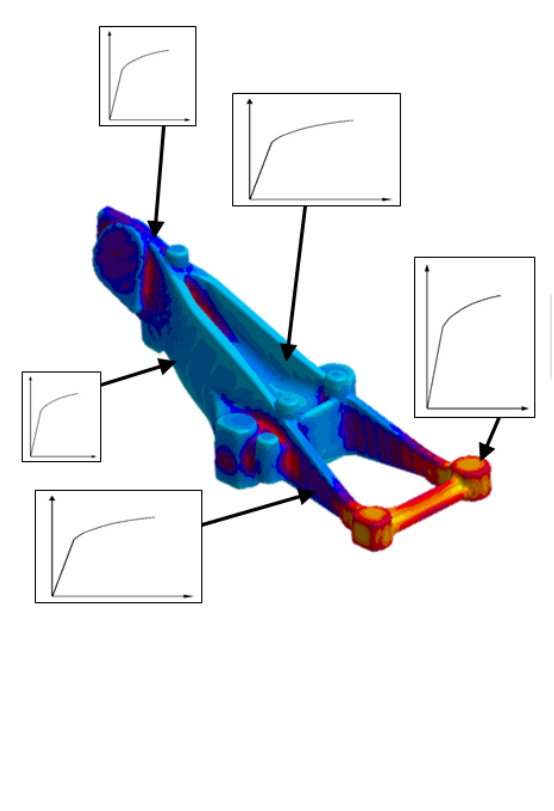

Fig. 11: Prediction of material performance through export of local stress-strain curves $[17,20]$.

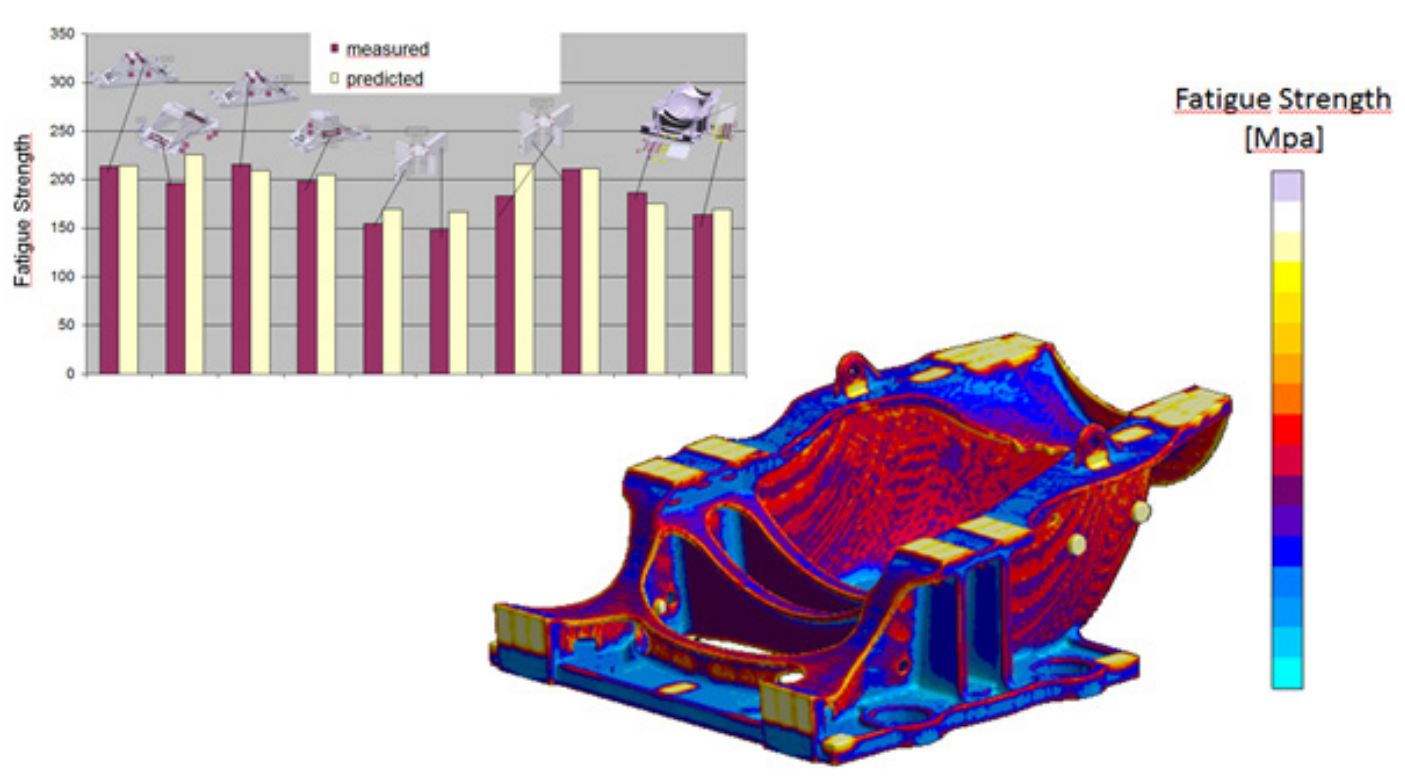

Fig. 12: Prediction and experimental validation of local fatigue strength as function of local structure predictions [12].

\section{Simulation Supports the Entire Manufacturing Route}

Aiming for a quantitative prediction of the final properties of the cast component when the part is shipped to the customer, casting process simulation must be able to address the entire manufacturing route of castings. In many cases, final component properties are determined by subsequent manufacturing steps, such as heat treatment. This is well known for white iron and malleable iron castings. In addition, the industrial application of austempered ductile iron, ADI, has grown in recent years. The material has a number of mechanical properties that makes it attractive for structural applications in industries such as automotive, heavy trucks, and many others. The material can be tailored to have properties such as high strength, high wear resistance, high fracture toughness, and high fatigue strength.

$\mathrm{ADI}$ is an alloyed ductile iron which has been subjected to a three-step austempering heat treatment process. The complex interaction of manufacturing conditions and microstructures is 
ideally suited to be assessed by process simulation. Knowing the local as-cast microstructure (nodule count, phase distribution, and segregation profiles), a coupled diffusion and kinetic model allows the simulation of the local formation of austenite and subsequent carbon pick-up as a function of time and heat treatment conditions, Fig. 13.

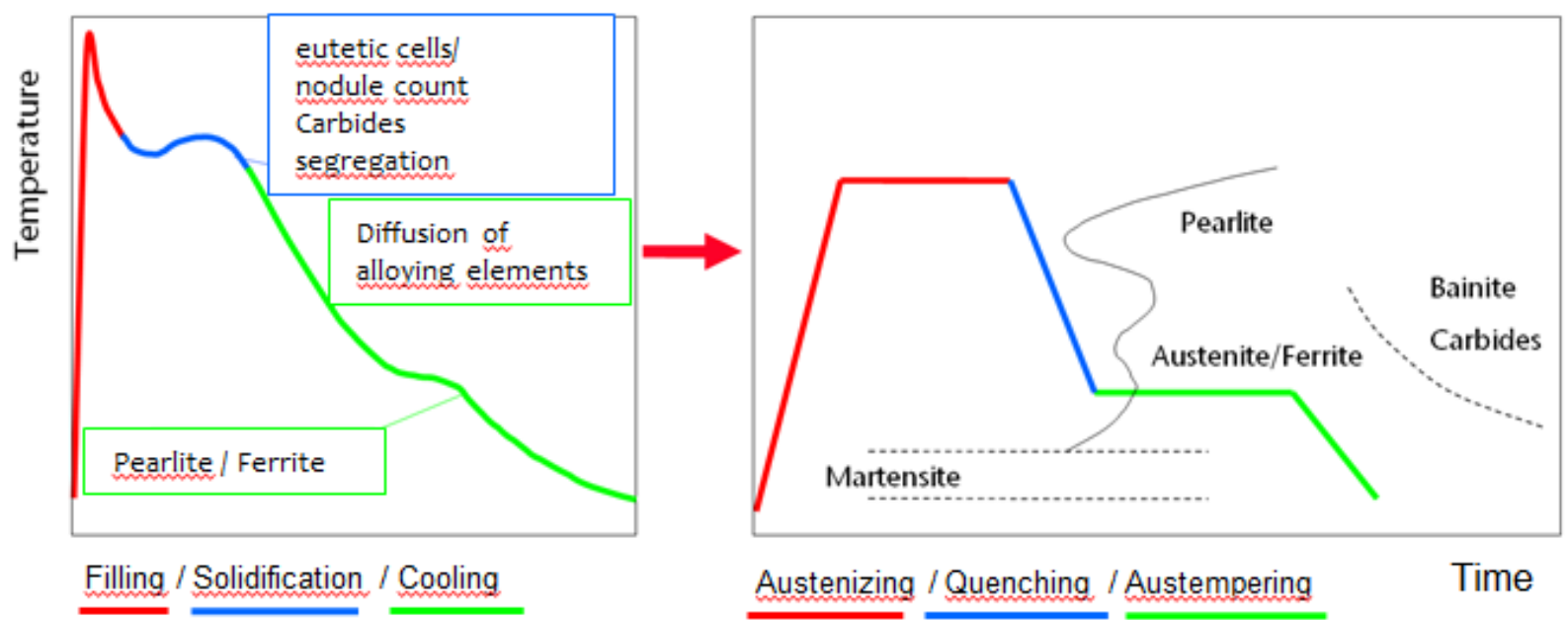

Fig. 13: Integration of casting process simulation and heat treatment simulation to predict ADI structures. The micromodelling of cast iron provides valuable information about structures and segregation profiles for a subsequent heat treatment simulation. Nodule count and segregation profiles are used as input values for the simulation of austenitization, subsequent quenching and austempering stages. The simulation provides quantitative information about microstructures at all stages of heat treatment, and allows determination of the required times to reach the respective structure [21].
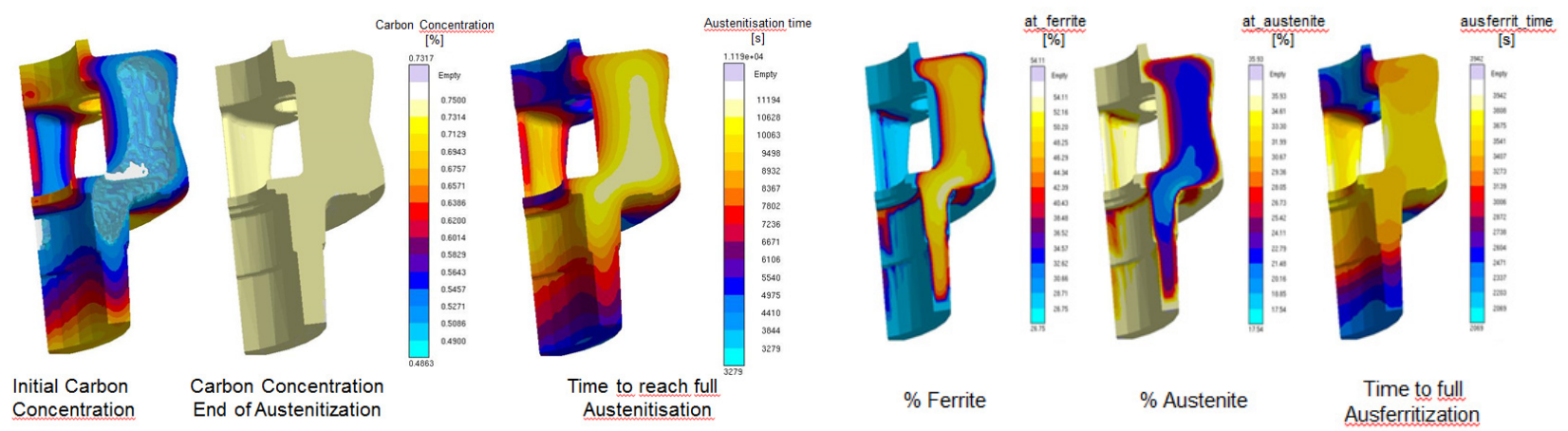

Fig. 14: Simulation of austenitization, quenching and ausferritization of a planetary carrier. Based on the local as-cast structure, the phase changes from solid-state phases into austenite, and the subsequent carbon saturation can be modeled. As a result, the carbon levels and the time to reach full saturation are predicted. Subsequently, nucleation and growth of ferrite and diffusion of carbon into the austenite is modeled. This leads to a quantitative prediction of final phase distributions and the time to reach a full ausferritization [21].

Today complementary micromodeling applications are available to model heat treatment processes for cast irons such as pearlitization. Similar models allow prediction of as-cast and heat treated structures (carbides) for high chrome white irons.

\section{Supporting Casting Design for Performance}

Quantitative knowledge about local properties helps both the foundry specialist and the casting designer. The foundryman can set up a robust process guaranteeing the required specifications. The designer should make use of local properties for his design considerations to fully exploit the potential of the casting. This has strongly supported the development of new and innovative cast components, such as wind turbine castings. 
An optimal use of cast iron properties is only possible if the designer uses the potential of the material to its full extent. This regards both weight savings as well as design for optimal performance in use. For this purpose, casting designers are asking for clear design rules and tools to support the design of the component.

Besides the geometry, cast iron casting properties are dependent on defects, the graphite morphology, and the structure of the matrix. The chosen metallurgy and the process control are main influencing parameters for the resulting casting and its performance. This interdependency leads to uncertainty regarding the real casting properties a designer can count on. Therefore standard values for castings are applied to secure the minimum requirements. Until now, designers consider varying casting properties more as threat than as an opportunity [22].

This makes clear that a close coupling of casting process simulation with performance simulation is needed. The full use of the material potential can only be realized, if the real material properties resulting from the casting process are introduced for the load calculations of the designer, Fig. 15. Casting process simulation must answer questions which will be asked by both the foundry specialist and the designer. Therefore, it is important that simulation is able to predict cast iron material behavior not only qualitatively but also quantitatively.

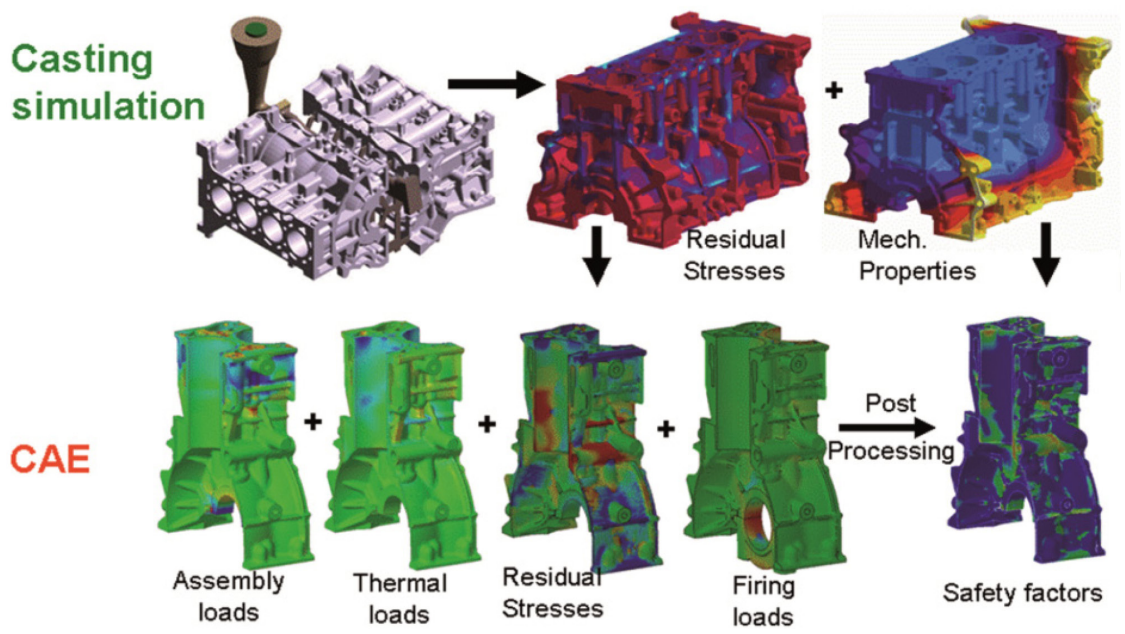

Fig. 15: Integrated CAE design-process chain. Only a coupled use of casting process simulation and performance simulation allows the assessment of the real material performance in a component with respect to its local mechanical properties and residual stresses [15] [16].

The integration of structure, defect, and property modelling of castings into the CAE world enables the designer to assess local mechanical performance for both the static and dynamic local behavior based on the real performance of the casting, Fig. 16. Alternatively, the material potential can be used for weight savings [23].

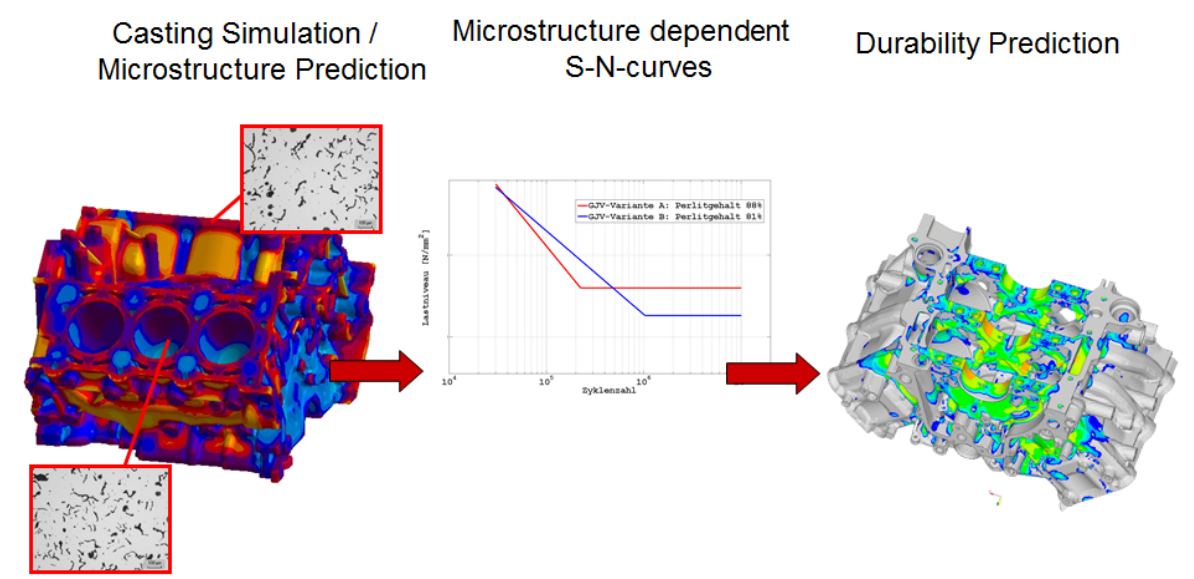

Fig. 16: Process of transferring casting structure and property distributions to lifetime prediction analysis [12] 


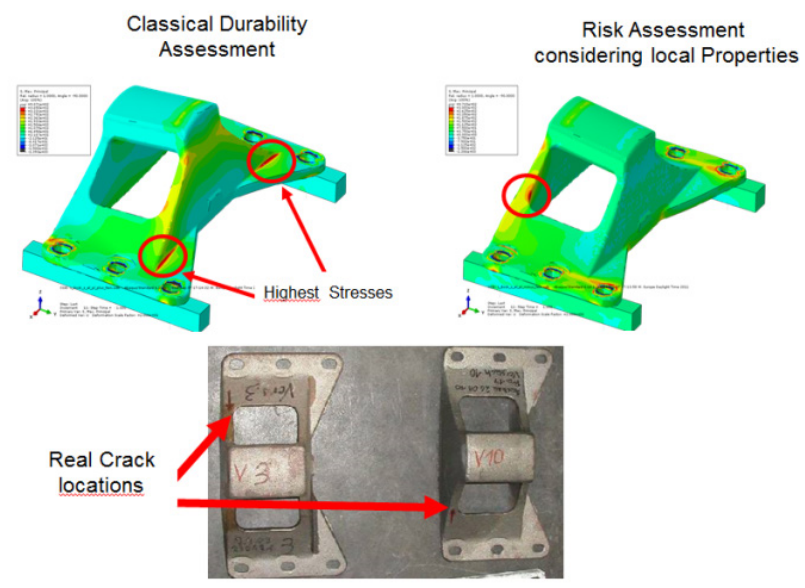

Fig. 17: Comparison of failure locations in a bearing support. Only with the consideration of local fatigue strength as a function of the local structures can the correct crack initiation locations be predicted [24].

\section{From Simulation to Optimized Solutions and Robust Process Lay-Out}

The evaluation of a robust and efficient manufacturing route is still one of the main objectives to use casting process simulation in a foundry. Due to the tight interaction of metallurgy and material properties, the foundry specialist still has open questions with respect to filling and solidification of cast iron castings. This is the case for a reproducible generation of the expected graphite morphology, the feeding performance and the resulting microstructures and properties.

Today's requirements on the development of a casting and the corresponding casting process demand methodologies and tools which allow a maximization of process robustness and profitability at the earliest possible point in time. Typically, the freedom to make improvements is in practice reduced to a small number of real trials during the development phase, and is limited by the effort connected with changing process parameters in series production. A quantitative estimate of real casting quality based on casting trials and a reduction of the required number of experiments to optimize the casting process remain a challenge.

Up to today, casting process simulation tools have been used by foundry engineers to confirm a set of selected process parameters and evaluate a given casting layout. They then make manual changes to process parameters or geometries, like runners, gates or feeders, to get closer to achieving the objectives they have in mind, and repeat this process until they find a satisfying solution, red/yellow curve in Fig 19.

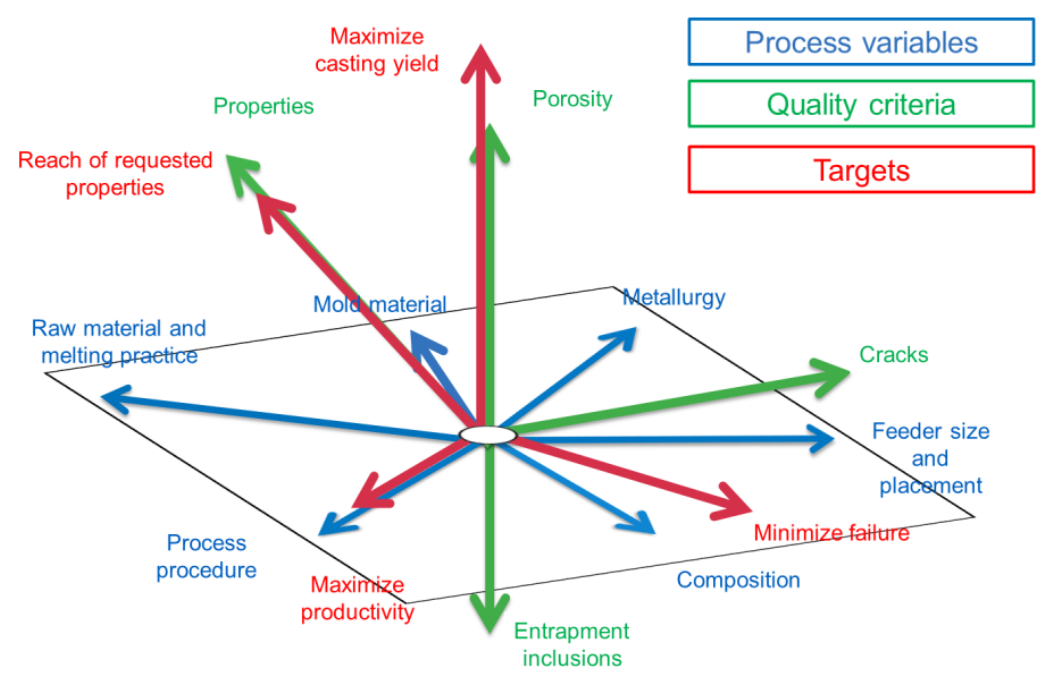

Fig. 18: The virtual design space for autonomous optimization is composed of varying process parameters, calculated quality criteria, and optimization targets defined in the software [25]. 
In contrast to real-world trials, the new methodology of virtual experimentation and autonomous optimization using simulation tools provide significantly more flexibility, especially for cast iron applications. Autonomous optimization enables engineers to vary several parameters, such as metallurgical conditions, chemical composition, and the casting process layout simultaneously and independently from each other. Different quality criteria can be evaluated individually and quantitatively, Fig. 18. Combined with established tools from statistical design of experiments, casting process simulation can be used to autonomously optimize casting processes and designs. The software follows several targets at the same time and finds the best compromise based on first principles, Fig 19 left. The automated assessment of all simulated quality criteria can be used to find the optimal route to achieve the desired objectives quickly and easily. In addition, the number of real-world trials can be reduced and the impact various process parameters have on reaching a robust process window can be assessed in early phases of casting, pattern making, and process development, Fig 19 right [26], [27].

The new methodology of autonomous optimization is not a replacement for process knowledge and expertise. Based on the technical and economical boundary conditions for his process, the foundry engineer needs to specify the parameters he has the flexibility to change considering the requirements placed on the casting and the objectives to be achieved. These objectives are made measureable by relating them to corresponding quality criteria. The questions to be addressed to the software are simple: What characterizes a good gating system (Fig. 20)? How do I accomplish a robust process window? How do I select process conditions that provide the required casting quality? Quantitative descriptions of the important influencing factors, measureable quality and cost indicators, and the goals to be achieved are required to answer these questions, Fig.21.
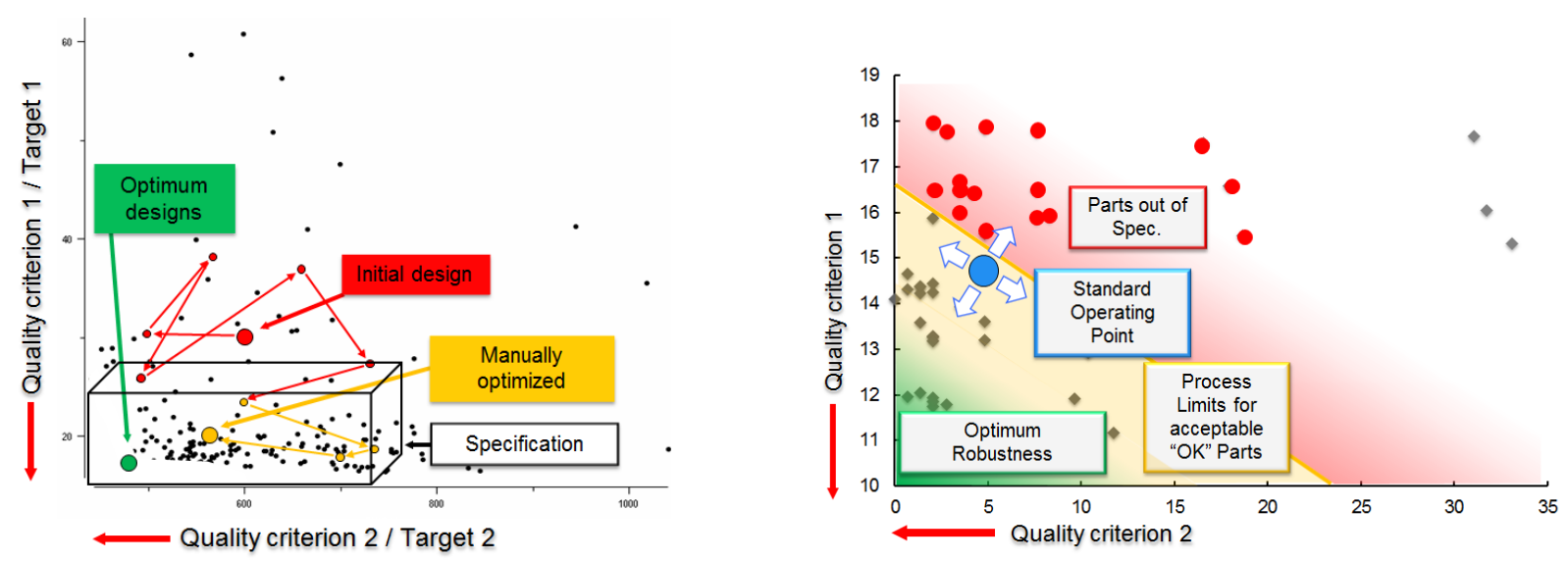

Fig. 19: Main goals using virtual experimentation and autonomous optimization: reaching the best compromise and optimum working conditions for different quality criteria (left) and establishing robust processes for standard operating conditions (right). Each of the marks in the charts represents an individual virtual experiment under different process conditions. 


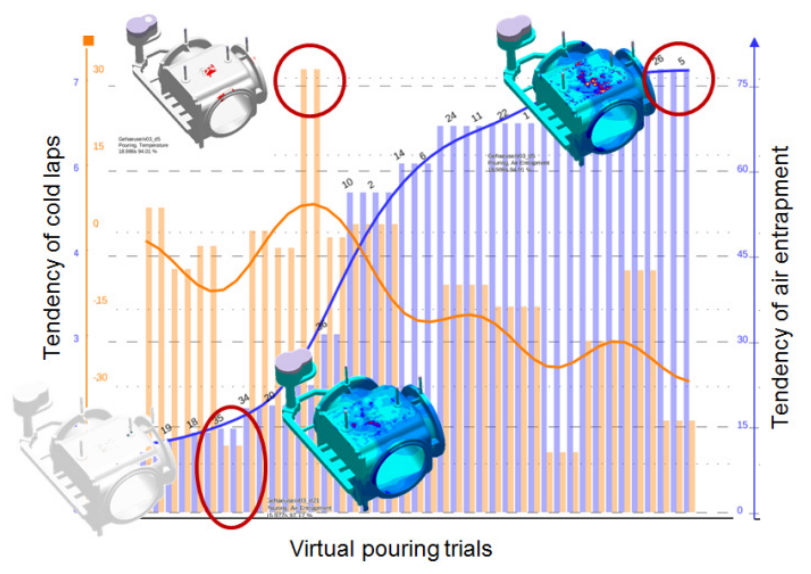

Fig. 20: Virtual experimentation evaluating different gating systems for the competing situation of cold laps and air entrapment leads to a distinct robust process window, in which both quality criteria are met (red circle).

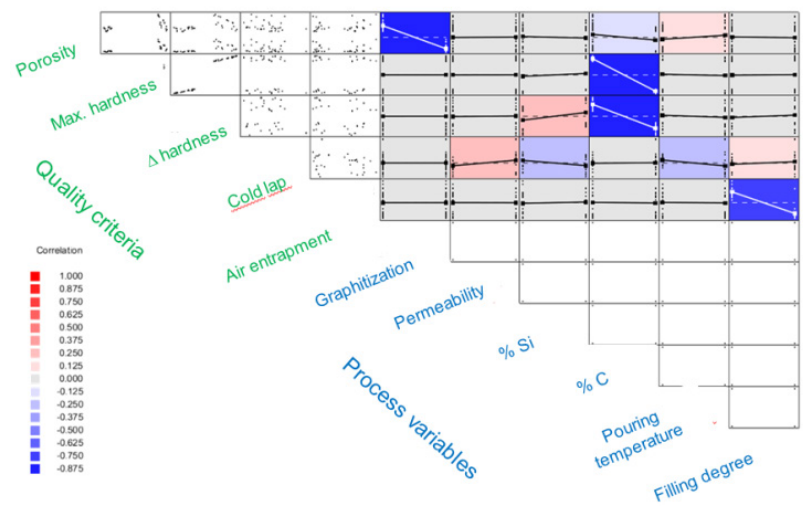

Fig. 21: Correlation diagram based on a virtual DOE allows the assessment of process variables and resulting quality. Blue and red charts indicate main effects between process variables and resulting quality.

\section{Summary}

The beauty of the casting process, realizing a complex component "in one pour" becomes a challenge, especially for cast iron due to the numerous interactions between different quality determining parameters. A simulation tool has to meet this challenge, especially with respect to the complexity of cast iron solidification. Only if a virtual tool considers the degrees of freedom the foundry specialist has to manufacture sound castings, can the foundryman benefit from the software for daily process and production optimization in his foundry.

The main goals of a foundry to use a casting process simulation tool - reproducible quality, increased profitability, adequate design for manufacture and entering into new markets - strengthen the competitiveness of the casting process as such. In this context, "casting quality" means more than "soundness", "cost reduction" means more than "improved yield", and "casting properties" mean more than "meeting required standards". The information provided by state-of-the-art technology supports both foundryman and casting user in achieving a design considering the local material and process demands for cast iron, as well as supporting the foundry specialist in setting up optimized and robust manufacturing conditions.

Since the early 1980's Ingvar Svensson has dedicated his impressive scientific career to understand how cast iron metals solidify and how they form their diversified structures and properties. But even more than this, Ingvar has always strived to pass on this knowledge to students and the foundry industry as a whole in a quantitative manner.

Not least due to Ingvar's outstanding work, casting designers as well as foundry experts benefit from better process understanding by using single simulations, virtual DOEs or autonomous optimizations. This allows them to establish robust casting designs and processes and produce quality castings at the lowest possible cost. It makes cast iron a predictable material for the future.

\section{References}

[1] P. N. Hansen, E. Flender and J. C. Sturm, Casting Process Simulation - From the Idea 30 Years ago to Reality Today, International Foundry Research 61 (2009) 12-29.

[2] J. C. Sturm, Die Prozess-Entwicklungs-Kette: Nutzung von Eigenschafts-Vorhersagen für Gusseisenwerkstoffe für innovative Bauteil-Konstruktionen, Gießerei 90 (2003) 56-58.

[3] J. C. Sturm, G. Busch, Cast Iron - a predictable material, WFC 2010 Hangzhou, China. 
[4] M. Schneider, J. C. Sturm, W. Schaefer, E. Hepp, V. Gurevich, Integrated Computational Materials Engineering and Modelling of Shape Casting Processes - Needs, Benefits, Limitations, and Hurdles, in Proceedings of MCWASP 2015 (21.-26.06.2015, Hyogo, Japan)

[5] H. Fredriksson, I .L. Svensson, Computer Simulation of the Structure Formed During Solidification of Cast Iron, The Physical Metallurgy of Cast Iron; Stockholm; Sweden; 29-31 Aug. 1984, pp. 273-284

[6] E. Lundbäck, I. L. Svensson, Prediction of Properties of Nodular Cast Iron Castings, by means of Computer Simulation, Proceedings of Modelling of Casting, Welding and Advanced Solidification Processes Conference TMS-V, Davos Sept. 1990

[7] M. Wessen, I. L. Svensson, Modeling of ferrite growth in nodular cast iron, Metall. Mater. Trans. A 27 (1996) 2209-2220.

[8] M. Wessén, I. Svensson (1999), Application of microstructure modelling for ductile iron alloy property optimization, Revue de Metallurgie, ISSN 0035-1563, 96 (no JA99) (1999) 142.

[9] I. L. Svensson, M. Wessén, Foundry of Cast irons: Processing and Simulation, Numerical Simulation of Foundry Processes, Sept. 2001, pp. 87-145

[10] A. Diószegi, I. L. Svensson, Inverse kinetic analysis method to study eutectic growth, Int. J. Cast Metal. Res. 18 (2005) 41-46.

[11] A. Diószegi, K. Liu, I. L. Svensson, Inoculation of Primary Austenite in Grey Cast Iron, Int. J. Cast Metal. Res. 20 (2007) 68-72.

[12] A. Heinrietz, J. Eufinger, W. Stets, u. a.: Maßgeschneiderte Bauteileigenschaften durch Integration von Fertigungs- und Funktionssimulation. Abschlussbericht BMBF Projekt Nr. 01R/0713, 2011.

[13] A. Rechsteiner, Proceedings of German MAGMA User Meeting, October 2003, Vaals, The Netherlands

[14] C. Heisser, J. C. Sturm, Casting Process Simulation of Compacted Graphite Iron, (03-025), 107th Casting Congress Milwaukee, Wisconsin, 26-28 April, 2003, pp. 685-692.

[15] U. Weiss, M. Broda, P. Rong, Die Rolle des Eisengießers bei der virtuellen Produktentwicklung im Automobilbau, Presentation on the MAGMA Seminar "Gusseisen Ein Werkstoff mit Zukunft", Duisburg, Mai 2002

[16] W. Simon, U. Weiss, Gussteilentwicklung im Zeichen neuartiger Anforderungen, Proceedings of NEWCAST-Forum, Düsseldorf: "Konstruieren mit Gusswerkstoffen”, June 18, 2003, pp. 3-11

[17] J. Olofsson, Simulation of Microstructure-based Mechanical Behaviour of Cast Components, Ph.D. thesis, (Jönköping University, School of Engineering, 2014)

[18] T. Sjögren, I. L. Svensson, Modelling the effect of graphite morphology on the modulus of elasticity in cast irons, Int. J. Cast Metal. Res. 17 (2004) 271-279.

[19] I. L. Svensson, T. Sjögren, On modeling and simulation of mechanical properties of cast irons with different morphologies of graphite, Inter. Metalcast. 3 (2009) 67-77

[20] I. L. Svensson, J. Olofsson, On microstructure-based mechanical behaviour of a ductile iron component, proceedings of 10th International Symposium on the Science and Processing of Cast Iron SPCI10, 2014, Mar det plata, Argentina

[21] E. Hepp, M. Dzusov, W. Schäfer, Process optimization for an energy efficient heat treatment of ADI, HTM J. Heat Treatm. Mat.70 (2015) 239-247 
[22] C. Thomser, M. Bodenburg, J.C. Sturm, Optimized durability prediction of cast iron based on local microstructure, Int. J. Metalcast. 11 (2017) 207-215

[23] J. Olofsson, I. L. Svensson, Incorporating predicted local mechanical behaviour of cast components into finite element simulations, Materials \& Design 34 (2012) 494-500

[24] C. Heisser, N. Zenker, E. Fritsche, J. C. Sturm, Prediction of microstructure and local mechanical properties for a CGI crankcase, Proceedings of the AFS Annual Congress, $06 / 2015$

[25] J.C. Sturm, I. Hahn, Von der Simulation zur gießtechnischen Optimierung, Giesserei 102 (2015) 86-100

[26] I. Hahn, J. C. Sturm, Autonomous optimization of casting processes and designs, WFO Technical Forum 2011, Düsseldorf, 28.06.-02.07.2011

[27] D. Coyle, Minimizing Casting Costs by Autonomously Simulating Different Gating Systems for Ductile Iron Castings, in Proceedings of the AFS Annual Congress 2017 